Extensions to three-dimensional flow in a porous channel

Hewitt, R.E. and Duck, P.W. and Al-Azhari, M.

2003

MIMS EPrint: 2013.70

Manchester Institute for Mathematical Sciences

School of Mathematics

The University of Manchester

\footnotetext{
Reports available from: http://eprints.maths.manchester.ac.uk/

And by contacting: The MIMS Secretary

School of Mathematics

The University of Manchester

Manchester, M13 9PL, UK
} 


\title{
Extensions to three-dimensional flow in a porous channel
}

\author{
R.E. Hewitt*, P.W. Duck, M. Al-Azhari \\ Department of Mathematics, University of Manchester, Oxford Road, Manchester M13 9PL, UK
}

Received 23 July 2002; accepted 2 December 2002

Communicated by T. Mullin

\begin{abstract}
We consider the flow of a viscous, incompressible fluid contained between two parallel, porous walls. The flow is driven by a spatially uniform injection/suction of fluid through the bounding walls. We extend the solution structure of previous investigations to a more general three-dimensional stagnation-point form which can capture a whole range of phenomena in a single class of states. In particular, we show that this form of solution contains states previously discussed under more restrictive assumptions on the flow field. We show that a range of two- and three-dimensional states exist, together with symmetry-broken solutions and periodic states. We discuss the stability of these states and relate the previous results of Drazin, Banks, Zaturska and co-workers to those of Goldshtik and Javorsky on the "bifurcation to swirl" and of Hewitt and Duck on non-axisymmetric von Kármán flows.

(c) 2003 Published by The Japan Society of Fluid Mechanics and Elsevier Science B.V. All rights reserved.
\end{abstract}

Keywords: Exact Navier-Stokes solutions; Symmetry breaking; Similarity solution; Berman problem

\section{Introduction}

The self-similar flow of a viscous, incompressible fluid driven by wall transpiration in a channel with parallel walls has been considered by many authors in recent years. Berman (1953) first posed a steady non-dimensional similarity form for the velocity field:

$$
\mathbf{u}(x, y, z, t)=(x U(z, t), W(z, t))^{\mathrm{T}}
$$

under the assumption of a mid-plane symmetry in the domain $z \in[-1,1]$, with $x$ a coordinate parallel to the channel walls. Here $U$ is a similarity component in the $x$-direction and $W$ is the component perpendicular to the bounding planes. The boundary conditions are those of no-slip $U( \pm 1)=0$ and uniform transpiration, with $W( \pm 1)= \pm 1$ for suction and $W( \pm 1)=\mp 1$ for injection.

\footnotetext{
* Corresponding author.

E-mail address: hewitt@ma.man.ac.uk (R.E. Hewitt).
} 
Berman (1953) gave the corresponding ordinary-differential reduction of the Navier-Stokes equations for this form of solution. The governing fourth-order equation is an exact reduction of the Navier-Stokes system and contains a single parameter, Re, which is a Reynolds number based on the suction/injection rate at the channel walls. Many authors have since extended the initial low Reynolds number series solutions of Berman.

Of particular relevance here are the sequence of papers by Zaturska et al. (1988), Watson et al. (1990) and Taylor et al. (1991). ${ }^{1}$ The first of these papers reconsidered the driven problem to include unsteady effects and showed that the primary symmetric branch loses stability to perturbations that break the mid-plane symmetry. Furthermore, it was shown that periodic states arise followed by a Lorenz-like transition to chaotic flows at higher suction/injection Reynolds numbers. The second paper in this sequence allowed for the combined effects of both transpiration and an accelerating wall motion (i.e., $U( \pm 1)= \pm U_{\mathrm{e}}$ ) via the inclusion of an additional parameter, $U_{\mathrm{e}}$, which represents the relative importance of suction and wall motion. The numerical results concentrated primarily on the case in which the transpiration was absent, showing that chaotic states arise through the mechanism of a period-doubling cascade. In the last of this sequence of papers, the original Berman flow was reconsidered (once again in the absence of any wall motion) with an extension towards three-dimensionality with solutions of the form

$$
\mathbf{u}(x, y, z, t)=(x U(z, t), y V(z, t), W(z, t))^{\mathrm{T}} .
$$

Note that (2) encompasses the classical stagnation-point flows of Hiemenz (1911) and Howarth (1934) (and the axisymmetric analogue of Homann, 1936).

At fixed Reynolds number, the solution structure is spanned by a single parameter, $\mu$, which essentially measures the flow's three-dimensionality. Two particular values of $\mu$ are of significance: $\mu=0$ corresponding to an axially symmetric radial flow (of which we shall discuss more later) and $\mu=1$ for which solutions of Berman-type form a subset of possible states. The exact nature of $\mu$ is discussed later in our formulation, since we shall adopt the same notation in our current work.

Further developments of this class of flow have been provided by Cox and King (1997), and Cox (1991) who demonstrated that the chaotic behaviour first described by Zaturska et al. (1988) was extremely sensitive to any asymmetry in the wall conditions, i.e., when the structure was unfolded with a small difference in the suction/injection rates at each boundary. More recently, Cox (2002) has considered three-dimensional extensions of the form presented by Taylor et al. (1991) in the case of one porous boundary and one impermeable boundary, together with fluid injection (corresponding to a floating disk on an air table). In this work, it was shown that (perhaps unsurprisingly given the results of Taylor et al. and the boundary-layer formulations of Davey and Schofield, 1967 and Hewitt et al., 2002) three-dimensional states are again possible as alternatives to the axisymmetric flows. The origin of these three-dimensional solutions and their development at low injection Reynolds numbers was not clarified, and we shall add to these results as a consequence of this paper.

It is perhaps worth noting that all the flow states mentioned above are "exact solutions" in the sense that numerical solutions of the resulting ordinary-differential system satisfy the Navier-Stokes system without approximation.

\footnotetext{
${ }^{1}$ Philip Drazin was a co-author of each investigation.
} 


\subsection{Related exact solutions}

Although the states described above are provided in a Cartesian framework, the corresponding axisymmetric solutions have been well studied. In a cylindrical polar coordinate system $(r, \theta, z)$, the class of non-dimensional von Kármán solutions exists

$$
\mathbf{u}(r, \theta, z)=(r \hat{U}(\theta, z, t), r \hat{V}(\theta, z, t), \hat{W}(\theta, z, t))^{\mathrm{T}}
$$

in a domain $z \in[-1,1]$.

As was observed by Hewitt and Duck (2000) and similarly by Hall et al. (1992) in a related context, for the similarity form (3) to be an exact reduction of the Navier-Stokes system one requires that the components either be independent of the azimuthal coordinate $\theta$, or of the special form

$$
\begin{aligned}
& \hat{U}(\theta, z, t)=\tilde{U}(z, t)+\Phi(z, t) \cos (2 \theta+\Delta), \\
& \hat{V}(\theta, z, t)=\tilde{V}(z, t)-\Phi(z, t) \sin (2 \theta+\Delta), \\
& \hat{W}(\theta, z, t)=\tilde{W}(z, t),
\end{aligned}
$$

where $\Delta$ is a constant. The connection between this latter exact, non-axisymmetric solution and the Cartesian description given above will be highlighted again later, but has already been discussed in the semi-infinite domain by Hall et al. (1992).

Descriptions of these states usually involve the use of impermeable rotating boundaries (the "rotating disk" configuration) but, given the form of (4c), are equally applicable to flows between uniformly porous boundaries in the absence of a forced swirl. Interestingly, the paper of Goldshtik and Javorsky (1989) (as part of a larger sequence regarding the phenomena of the generation of swirl) has shown that even without forcing rotation at the boundaries, swirl can be generated in the flow through a bifurcation at a critical rate of withdrawal of fluid through the bounding walls.

In the context of solution (2) above, it is clear that the swirl-free axisymmetric von Kármán states (i.e., $\tilde{V} \equiv \Phi \equiv 0$ in (4)) correspond to the case in which $U=V$ in the notation of (2), and therefore $\tilde{U}=U=V$ in (4). Furthermore, on resolving the non-axisymmetric state (4) into the Cartesian formulation one finds that the corresponding flow field (to within an arbitrary rotation; here we take $\Delta=0$ ) is

$$
\mathbf{u}(x, y, z, t)=\left(\begin{array}{c}
x[\tilde{U}(z, t)+\Phi(z, t)]-y \tilde{V}(z, t) \\
x \tilde{V}(z, t)+y[\tilde{U}(z, t)-\Phi(z, t)] \\
\tilde{W}(z, t)
\end{array}\right) .
$$

\section{Formulation}

We consider the flow of an incompressible fluid of kinematic viscosity $v$ confined between two parallel porous planes separated by a distance $2 h$ and driven by a suction/injection velocity $\Gamma_{\mathrm{w}}$ through the bounding walls. 
Given the above descriptions of previous solution types to the porous channel flow problem, we see that the work of Drazin, Banks and Zaturska can be further extended to solutions of the form

$$
\mathbf{u}(x, y, z, t)=\left(\begin{array}{c}
x f_{z}(z, t)+y F(z, t) / \sqrt{c} \\
x \delta F(z, t) \sqrt{c}+y g_{z}(z, t) \\
-f(z, t)-g(z, t)
\end{array}\right)
$$

for any positive constant $c$ with $\delta= \pm 1$, where $\{x, y, z\}$ is the Cartesian coordinate system, nondimensionalised with the natural lengthscale $h$ and $\mathbf{u}$ is the velocity field non-dimensionalised with respect to the suction/injection rate at the $z=-1$ boundary. In the notation of (6), subscripts are used to denote differentiation with respect to the subscript variable. Here the dimensionless pressure distribution is of the form

$$
p(x, y, z, t)=K_{1}(t) \frac{x^{2}}{2}+K_{2}(t) \frac{y^{2}}{2}+Q(z, t),
$$

where $K_{1,2}(t)$ and $Q(z, t)$ are pressure functions.

Substitution of (6) into the Navier-Stokes system and differentiation to eliminate $K_{1,2}(t)$ provides a governing system in the form

$$
\begin{aligned}
& f_{z z t}+\left(f_{z}-g_{z}\right) f_{z z}-(f+g) f_{z z z}+2 \delta F F_{z}=\frac{1}{R e} f_{z z z z}, \\
& g_{z z t}+\left(g_{z}-f_{z}\right) g_{z z}-(f+g) g_{z z z}+2 \delta F F_{z}=\frac{1}{R e} g_{z z z z}, \\
& F_{t}+f_{z} F+g_{z} F-(f+g) F_{z}=\frac{1}{R e} F_{z z} .
\end{aligned}
$$

Here, $R e=\Gamma_{\mathrm{w}} h / v$ is the Reynolds number based on the suction/injection velocity and the boundary conditions to be applied are no-slip

$$
f_{z}( \pm 1, t)=0, \quad g_{z}( \pm 1, t)=0, \quad F( \pm 1, t)=0
$$

and permeability of the bounding walls

$$
\begin{aligned}
& f(-1, t)= \pm \frac{1}{2}(1+\mu), \quad g(-1, t)= \pm \frac{1}{2}(1-\mu), \\
& f(1, t)=\mp \frac{\gamma}{2}(1+\mu), \quad g(1, t)=\mp \frac{\gamma}{2}(1-\mu)
\end{aligned}
$$

with the upper sign indicating suction applied at the boundaries, whilst the lower sign corresponds to injection (we take $\gamma \geqslant 0$ ). In this formulation we have adhered to the notation used in the sequence of papers by Drazin, Banks, Zaturska and co-workers when $\gamma=1$ and $F \equiv 0$.

We note that in this differentiated form of the equations, the pressure constants are absent and the solution structure is parameterised by $\mu$ at a given $R e$ and $\gamma$. There are clearly two outward mass flux components; $M^{(x)}$ across a plane at a constant $x$ location (with $y \in[-l / 2, l / 2]$ ) and $M^{(y)}$ across a plane at a constant $y$ location (with $x \in[-l / 2, l / 2]$ ). It is easy to see from the form of (6) that

$$
M^{(x)}=\mp l(1+\mu)\left(\frac{1+\gamma}{2}\right) x, \quad M^{(y)}=\mp l(1-\mu)\left(\frac{1+\gamma}{2}\right) y .
$$


Here, as above, the upper sign indicates withdrawal of fluid through the boundaries and the lower sign indicates injection of fluid. Thus we see that specification of the parameter $\mu$ fixes the relative mass flux in the two perpendicular directions parallel to the bounding planes.

We note that there are some obvious sub-cases to this general formulation: (i) $\gamma=1, \mu=0$ admits solutions to the mid-plane-symmetric suction/injection flow that are axisymmetric, although as we shall see, other more general states are possible; (ii) $\gamma=1, \mu=1$ admits solutions of two-dimensional Berman-type, although again, more general states will be seen to exist; (iii) $\gamma=0$ corresponds to one boundary being impermeable, for which solutions are again available for $\mu \in[0, \infty)$. (As we have discussed in the introduction, some of these cases have been described in the existing literature, we do not repeat the full set of citations here.)

As a final note regarding this formulation, we note that the distribution of the pressure is determined a posteriori, thereby determining the functional dependence

$$
\frac{K_{1}(t)}{K_{2}(t)}=\frac{\operatorname{Re}[f(0, t)+g(0, t)] f_{z z}(0, t)+f_{z z z}(0, t)}{\operatorname{Re}[f(0, t)+g(0, t)] g_{z z}(0, t)+g_{z z z}(0, t)} .
$$

In the analysis of Cox (2002) for the injection flow between a porous boundary and an impermeable plane $(\gamma=0)$, the steady solutions are constructed subject to the condition that $K_{1} \equiv K_{2}$, therefore the solution structure of the Cox analysis corresponds to a cross-section of the $\{\mu, R e\}$ parameter space presented here.

\section{The (steady) floating disk problem $(F=0)$}

\subsection{Axisymmetric pressure distributions $\left(K_{1}=K_{2}\right)$ with non-axisymmetric mass flux}

Here we consider the steady flow between a permeable table (at $z=-1$ ) and an impermeable disk (at $z=+1$ ), in particular for solutions with axisymmetric pressure distributions, i.e. those with $K_{1}=K_{2}$. The axisymmetric version of this problem has been considered previously by Hinch and Lemaître (1994) and later for non-axisymmetric alternative solutions by Cox (2002).

The solutions presented by Cox are formulated under the condition that the pressure is axisymmetric (i.e., $K_{1}=K_{2}$ ). Cox examines solutions of this restricted form based on the tacit assumption that in the flow under any finite disk, the transition region around the edge of the disk acts to impose an axisymmetric pressure on the inner (self-similar) solution. Obviously without a detailed investigation of any outer edge region, we cannot say if more general forms of solution are available in which $K_{1} \neq K_{2}$ in the inner region and this pressure non-axisymmetry is removed in the outer edge region to match with the uniform surrounding atmospheric conditions.

Nevertheless, we can elucidate some of the remarks made by Cox regarding the solution structure for $K_{1}=K_{2}$. In terms of our formulation herein there is a locus of points in the $R e-\mu$ plane at which $K_{1}=K_{2}$. Clearly, along $\mu=0$ the mass flux in both directions is equal and the solution is a Cartesian description of an axisymmetric state more readily addressed in terms of a cylindrical-polar coordinate system. However, there are other instances in which an axisymmetric pressure distribution can be achieved even with non-axisymmetric mass flux. These states correspond to solutions for which $K_{1}=K_{2}$ but $f^{\prime}(z) \neq g^{\prime}(z)$ and occur on a locus of points in the $\mu-R e$ parameter plane. 


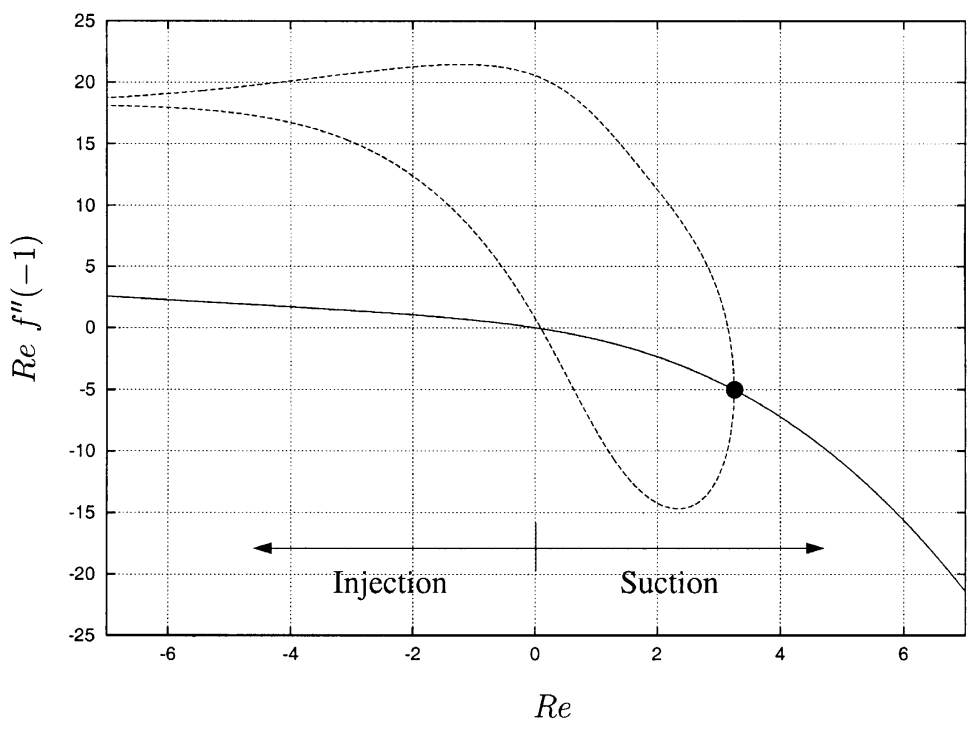

Fig. 1. The appearance of a non-axisymmetric injection state (shown as a dashed line) through a sub-critical pitchfork bifurcation in the suction regime and its continuation (through a singular solution in the low-Re limit) to the injection flow regime. Note that the two branches of the pitchfork correspond to an interchange of $f$ and $g$.

These non-axisymmetric states have already been described by Cox (2002), however there was no detailed discussion regarding their stability and how they arose. Indeed, Cox was unable to continue the solutions to lower Reynolds numbers and noted that "the reason why the calculation cannot be continued for $R e<22$ is unclear". (Note that the Reynolds number referred to by Cox is positive for injection flows and based on the gap width, whereas ours is negative for injection flows and based on the half-gap width.) Based on our numerical approach we can address both the origin and the stability of the non-axisymmetric states.

As shown in Fig. 1, the non-axisymmetric states referred to by Cox, for which $f^{\prime} \neq g^{\prime}$, arise through a sub-critical pitchfork bifurcation. The axisymmetry is lost at a (suction) Reynolds number of approximately 3.2517, with the nonlinear (sub-critically) bifurcated state continuing to negative (injection) Reynolds numbers and persisting in the limit of large injection rates $(\operatorname{Re} \rightarrow-\infty)$.

On the basis of these results, we may predict (and have confirmed via an appropriately formulated initial-value problem) that the non-axisymmetric solution branch is unstable as formulated by Cox (2002).

The non-axisymmetric state in the low Reynolds number limit is (formally) singular with

$$
f(z)=\hat{f}(z) R e^{-1}, \quad g(z)=\hat{g}(z) R e^{-1} \quad \text { as } R e \rightarrow 0 .
$$

In the limit $R e \rightarrow 0$, the leading-order form $(\hat{f}, \hat{g})$ of the non-axisymmetric solution satisfies the full equations (7), but with $R e$ replaced by unity and the boundary conditions are homogeneous (there being no injection/suction in this limit). Clearly, the trivial solution exists when $\operatorname{Re} \rightarrow 0$ and it is from this state that the axisymmetric solution branch originates; however there is essentially a nonlinear eigenfunction solution as demonstrated in Fig. 2, and it is from this limiting solution that the non-axisymmetric branch may be continued. Although the solution is "singular" in the above 


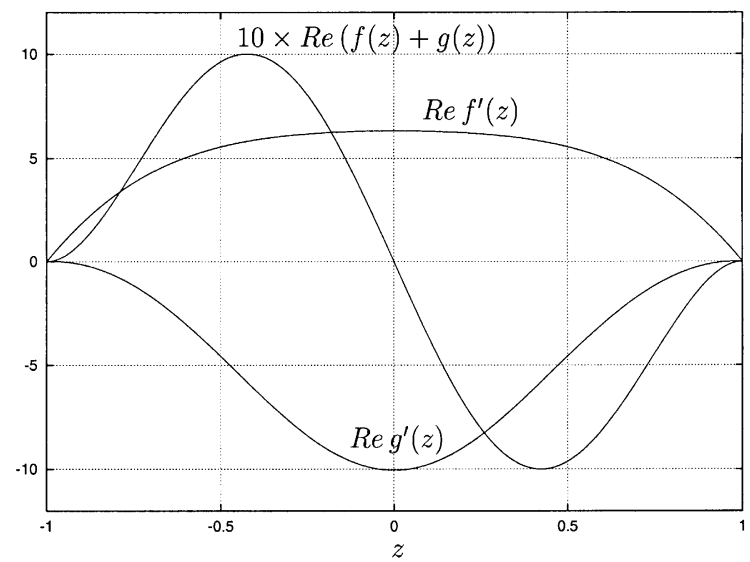

Fig. 2. The leading-order solution of the low-Reynolds non-axisymmetric state. Note that this is one of the non-trivial $R e=0$ states shown in Fig. 1, the other state simply corresponds to an interchange of $f$ and $g$.

context, we note that there is no physical singularity and the asymptotic form above arises from the definition of the suction rate as the natural velocity scale in the Reynolds number.

\subsection{Non-axisymmetric pressure distributions $\left(K_{1} \neq K_{2}\right)$}

We next consider flows with non-axisymmetric pressure distributions, i.e. for which $K_{1} \neq K_{2}$, but still such that $F=0$; a point of interest is that such solutions do not appear to exist for all values of the parameter $\mu$ in general. Fig. 3 shows the variation of (lower) wall shear stresses at $R e=20$ as the value of $\mu$ increases, and very strongly hints at the formation of a finite- $\mu$ singularity, and further (convincing) proof of this can be gleaned by the behaviour of the flow profiles of $f(z)$ and $g(z)$, as shown in Figs. 4 and 5, respectively. These latter figures show the respective profiles at increasing values of $f^{\prime \prime}(-1)$ (and hence $\mu$ ) as indicated; it is clear that: (i) $f(z)$ takes on a very pronounced, large amplitude sinusoidal profile, whilst $g(z)$ takes on (an equally pronounced) linear form, and is generally $O(1)$ in magnitude. This type of behaviour was observed in many calculations in this regime, and indicates that breakdown occurs at a critical value of $\mu$, say $\left(\mu_{\mathrm{c}}\right)$. These (and other) computations suggest that as $\mu \rightarrow \mu_{\mathrm{c}}$, the solution takes on the following form in the core of the flow, away from the walls

$$
\begin{aligned}
& f(z)=\left(\mu_{\mathrm{c}}-\mu\right)^{-2} f_{0}(z)+\left(\mu_{\mathrm{c}}-\mu\right)^{-1} f_{1}(z)+\cdots, \\
& g(z)=g_{0}(z)+\left(\mu_{\mathrm{c}}-\mu\right) g_{1}(z)+\cdots, \\
& K_{1}=\left(\mu_{\mathrm{c}}-\mu\right)^{-4} K_{10}+\left(\mu_{\mathrm{c}}-\mu\right)^{-3} K_{11}+\cdots, \\
& K_{2}=K_{20}+\left(\mu_{\mathrm{c}}-\mu\right) K_{21}+\cdots .
\end{aligned}
$$

Substitution of these into system (20b) leads us immediately to conclude that

$$
g_{0}(z)=\frac{1}{2} \Lambda_{+}(z+1)+\frac{1}{2} \Lambda_{-}(1-z)
$$




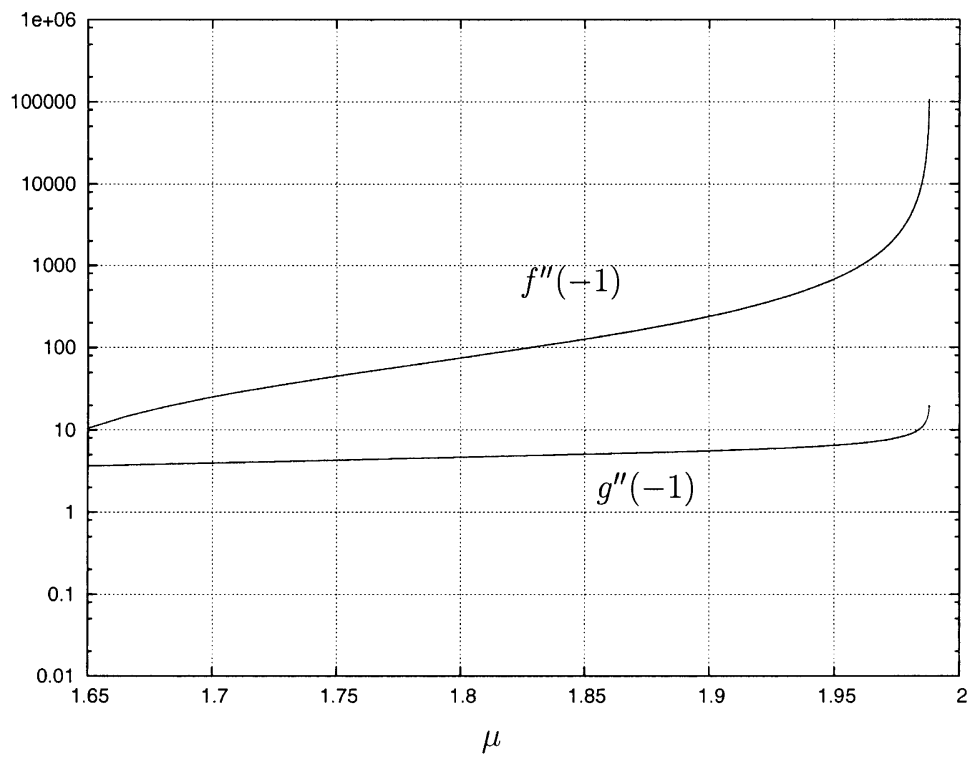

Fig. 3. Development of $f^{\prime \prime}( \pm 1)$ and $g^{\prime \prime}( \pm 1)$ for varying $\mu$ with $\gamma=0, R e=20, \delta=0$. The calculations begin on the solution branch that may be continued to the low-Reynolds number limit.

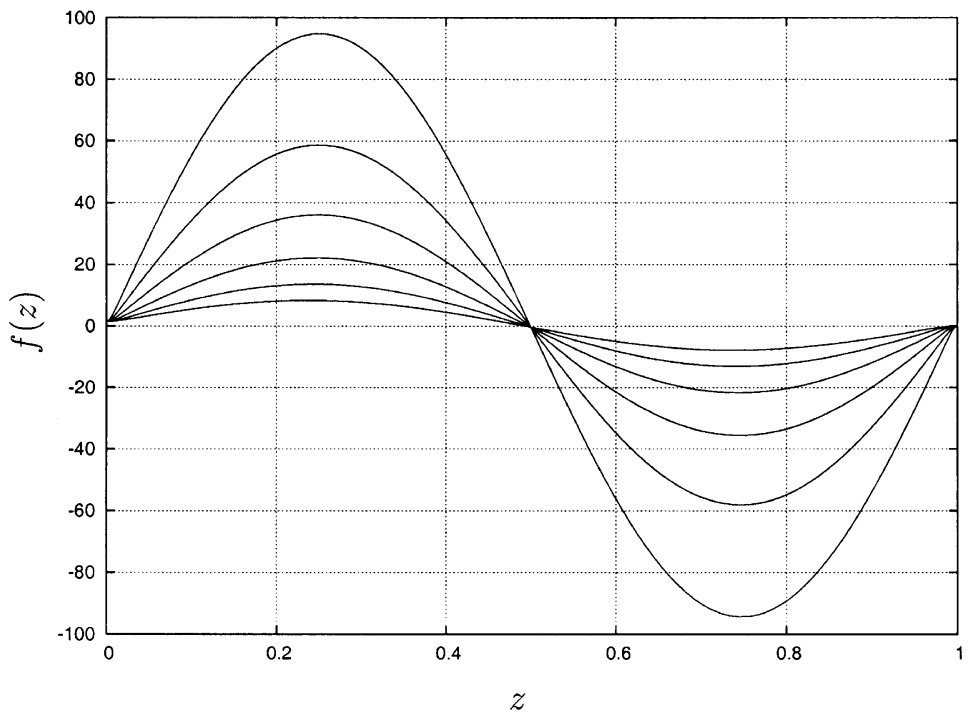

Fig. 4. $f(z)$ profiles for $f^{\prime \prime}(-1)=1000,2000,4000,8000,16,000,32,000(\mu=1.960756,1.973276,1.980298,1.984152$, $1.986205,1.987244$, respectively), on the solution branch of Fig. 3 with $\gamma=0, \operatorname{Re}=20, \delta=0$.

where $g( \pm 1)=\Lambda_{ \pm}$as determined by (8) above, evaluated at $\mu=\mu_{\mathrm{c}}$. Note also this serves to determine $K_{20}$, namely

$$
K_{20}=-\frac{1}{4}\left(\Lambda_{+}-\Lambda_{-}\right)^{2} .
$$




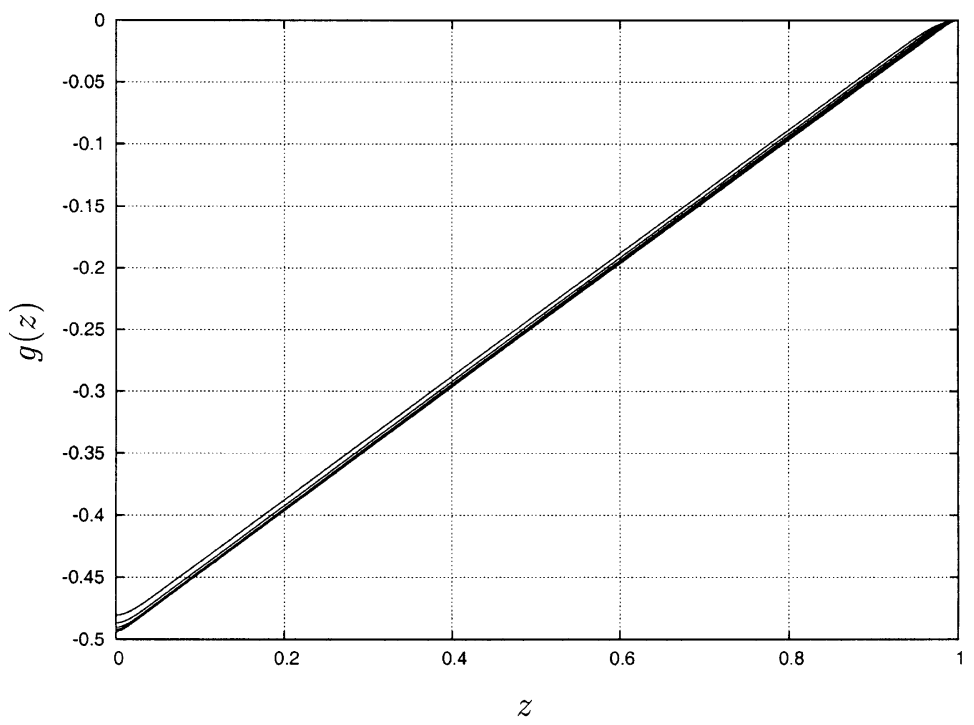

Fig. 5. $g(z)$ profiles for $f^{\prime \prime}(-1)=1000,2000,4000,8000,16000,32,000 \quad(\mu=1.960756,1.973276,1.980298,1.984152$, $1.986205,1.987244$, respectively), on the solution branch of Fig. 3 with $\gamma=0, \operatorname{Re}=20, \delta=0$.

$f_{0}(z)$ is determined from the $O\left(\left(\mu_{\mathrm{c}}-\mu\right)^{-4}\right)$ terms in $(20 \mathrm{~b})$ which are

$$
f_{0} f_{0 z z}-f_{0 z}^{2}=K_{10}
$$

which leads us to conclude (implementing the "reasonable" boundary conditions of impermeability at this order) that

$$
f_{0}(z)=\frac{\left(-K_{10}\right)^{1 / 2}}{n \pi} \sin (n \pi z)
$$

which is entirely consistent with the form observed in our numerical results, which strongly point to the case $n=1$, although there appears to be no reason why other integer values are not possible. Clearly, neither (13) nor (16) satisfy the no-slip conditions on the walls, necessitating the need for wall layers (on both walls). Taking the lower wall layer (for example), then the asymptotic form is

$$
\begin{aligned}
& f=\left(\mu_{\mathrm{c}}-\mu\right)^{-1} f_{0}^{*}\left(z^{*}\right)+f_{1}^{*}\left(z^{*}\right)+\cdots, \\
& g=\Lambda_{-}+\left(\mu_{\mathrm{c}}-\mu\right) g_{1}^{*}\left(z^{*}\right)+\cdots,
\end{aligned}
$$

where the boundary-layer variable $z^{*}=\left(\mu_{\mathrm{c}}-\mu\right)^{-1}(z+1)$. The equation determining $f_{0}^{*}$ is then

$$
\frac{1}{R e} f_{0 z^{*} z^{*} z^{*}}^{*}+f_{0}^{*} f_{0 z^{*} z^{*}}^{*}-f_{0 z^{*}}^{* 2}=K_{10}
$$

subject to

$$
f_{0}^{*}(0)=f_{0 z^{*}}^{*}(0)=0, \quad f_{0 z^{*}}^{*} \rightarrow\left(-K_{10}\right)^{1 / 2} \text { as } z^{*} \rightarrow \infty .
$$

The layer on $z=1$ is a mirror image of this layer. 
It should be noted that although in the above computation, $f(z)$ exhibits the pronounced singular behaviour in the core, it is possible to make choices of the parameters $\mu$ and $\gamma$ for which $g(z)$ would be the flow quantity exhibiting this feature (with $f(z)$ taking on the aforementioned linear form in the core); in this case the expansions for $f(z)$ and $g(z)$ become interchanged.

The conclusion, therefore, is that no steady-state solutions exist beyond $\mu_{\mathrm{c}}$ (dependent upon $R e$ ), at least in a local sense. It does appear, however, that in the limit of zero Reynolds number, i.e., $R e \rightarrow 0$, that solutions $d o$ exist for all $\mu$, and our numerical results (not presented here) firmly pointed to $\mu_{\mathrm{c}}$ being inversely proportional to $R e$ in this limit.

As a final note, we may point out that these numerical solutions (and those to follow) were derived by the application of four independent numerical algorithms, including both steady and unsteady central-differenced approaches that apply Newton iteration, an unsteady spectral scheme, and also using the (steady) bifurcation and continuation library of AUTO.

\section{Solutions with $F \neq 0$}

In this section, we consider solutions of the form (6) for which the additional component $F$ is non-zero. We begin by noting that integrating the steady form of the equations for $f(z)$ and $g(z)$ in (7) provides

$$
\begin{aligned}
& \frac{1}{R e} f_{z z z}+(f+g) f_{z z}-f_{z}^{2}-\delta F^{2}=K_{1}, \\
& \frac{1}{R e} g_{z z z}+(f+g) g_{z z}-g_{z}^{2}-\delta F^{2}=K_{2} .
\end{aligned}
$$

It is clear that one may introduce the new dependent variables

$$
\Psi(z)=f(z)-g(z), \quad \Gamma(z)=f(z)+g(z),
$$

satisfying

$$
\begin{aligned}
& \frac{1}{R e} \Psi_{z z z}+\Gamma \Psi_{z z}-\Psi_{z} \Gamma_{z}=K_{1}-K_{2} \\
& \frac{1}{\operatorname{Re}} \Gamma_{z z z}+\Gamma \Gamma_{z z}-\frac{1}{2}\left(\Gamma_{z}^{2}-\Psi_{z}^{2}\right)-2 \delta F^{2}=K_{1}+K_{2} .
\end{aligned}
$$

The boundary conditions for a symmetric suction flow (i.e., between two equally permeable boundaries, with $\gamma=1$ ) are then

$$
\Gamma_{z}( \pm 1)=\Psi_{z}( \pm 1)=F( \pm 1)=0, \quad \Gamma( \pm 1)=\mp 1, \Psi( \pm 1)=\mp \mu .
$$

From Eqs. (22a) and (7c), it is apparent that a class of non-trivial $F$ states may be obtained. In particular, if we take $K_{1}=K_{2}$ (i.e., an axisymmetric pressure distribution, though this does not necessarily imply an axisymmetric velocity field) then the governing system is formed from (22b) together with

$$
\mathscr{L}\left\{\Psi_{z}\right\}=0, \quad \mathscr{L}\{F\}=0,
$$


where the quasi-linear differential operator $\mathscr{L}$ is defined by

$$
\mathscr{L} \equiv \frac{1}{R e} \frac{\mathrm{d}^{2}}{\mathrm{~d} z^{2}}+\Gamma \frac{\mathrm{d}}{\mathrm{d} z}-\Gamma_{z}=0 .
$$

We may thus write $F=\alpha \hat{F}, \Psi_{z}=\beta \hat{F}$, for constants $\alpha$ and $\beta$, where $\mathscr{L}\{\hat{F}\}=0$ and $\hat{F}$ is arbitrarily normalised such that $\hat{F}_{z}(-1)=1$.

Given this solution, Eq. (22b) becomes

$$
\frac{1}{\operatorname{Re}} \Gamma_{z z z}+\Gamma \Gamma_{z z}-\frac{1}{2} \Gamma_{z}^{2}-\hat{F}^{2}\left(\frac{\beta^{2}}{2}-2 \delta \alpha^{2}\right)=2 K_{1},
$$

coupled to $\mathscr{L}\{\hat{F}\}=0$ with boundary conditions $\Gamma_{z}( \pm 1)=0, \Gamma( \pm 1)=\mp 1$, and $\hat{F}( \pm 1)=0, \hat{F}_{z}(-1)=1$. In this formulation, because of the form of the nonlinear coupling, at a given Re a solution can only be found at a fixed value of the coupling coefficient $\beta^{2} / 2-2 \delta \alpha^{2}$. Clearly, this only acts to determine the amplitude of the entire coupling term rather than $\alpha$ and $\beta$ individually. Thus, a continuum of solutions exist in which the influence of the $F$ term is parameterised by the amplitude coefficient $\alpha$. We note further that the mass flux parameter $\mu$ does not play a direct rôle in this formulation; however, for computational solutions of the original system cast into the description above we find that $\mu$ effectively determines $\alpha, \beta$ and parameterises the same continuum of states.

Since the pressure distribution is axisymmetric for this class of solution, a more easily interpreted formulation can be given in the context of the Hewitt and Duck (2000) and Al-Azhari (2003) discussions. Resolution of the above solution into a cylindrical polar coordinate system yields a von Kármán-like velocity field $(r \hat{U}, r \hat{V}, \hat{W})^{\mathrm{T}}$ relative to $(r, \theta, z)$ in the form given by (4) where $U^{\sim}=\Gamma_{z} / 2$, $V \stackrel{\tilde{=}}{=} A \Psi_{z} / 2, W \stackrel{\tilde{=}}{=}-\Gamma$, and $\Phi=B \Psi_{z} / 2$. Furthermore, the nonlinear coupling is such that only the combination $B^{2}-A^{2}$ is determined, and the individual values of $A$ and $B$ are left free to parameterise a continuum of states.

If $B=0$, i.e., there is axisymmetry since $\Phi \equiv 0$, then the solutions are those provided by Goldshtik and Javorsky (1989), leading to a swirl state through a supercritical pitchfork bifurcation.

When $A=0$, the flow bifurcates sub-critically to a non-axisymmetric solution with no swirl, this is the same solution that we continued in Section 2 (although in that section we applied $\gamma=0$, for an impermeable upper boundary) to illustrate the origin of the non-axisymmetric "air-disk" flows reported by Cox (2002).

Furthermore, the supercritical nature of the $B=0$ states and sub-critical nature of the $A=0$ states are again easily seen in this formulation since the sign of the all important nonlinear coupling term is inverted by switching between these two cases. In other words, one can derive a Stuart-Landau equation in a straightforward manner that is valid in the neighbourhood of the pitchfork bifurcation and in which the nonlinear cubic term changes sign in the two cases $A=0$ and $B=0$.

A continuum of states exists between these two extremes in which non-axisymmetry and swirl can be combined in arbitrary ratios although the combination $B^{2}-A^{2}$ must remain fixed. In the cylindrical polar formulation, it is the ratio of $B / A$ that parameterises the continuum of solutions; however, in the formulation of (6) this continuum is parameterised by the mass flux parameter $\mu$, with the case $B=0$ occurring at $\mu=0$ when $c=1$ (axisymmetric mass flux).

At fixed values of $\mu$ with $\delta=-1$, the bifurcation diagram is shown schematically in Fig. 6 . States of non-zero $F$ arise via the supercritical pitchfork bifurcation, with the appearance of periodic 


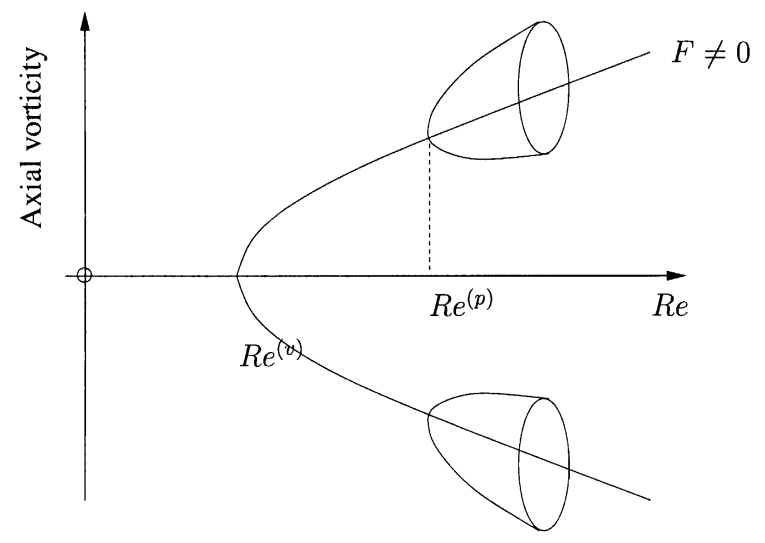

Fig. 6. The bifurcation diagram for the transition to states with non-zero $F$ (equivalent to the generation of axial vorticity) for a fixed $\mu$. Here, $R e^{(\mathrm{v})}$ denotes the pitchfork bifurcation to states of non-zero axial vorticity, whilst $R e^{(\mathrm{p})}$ indicates the secondary instability via supercritical Hopf bifurcation.
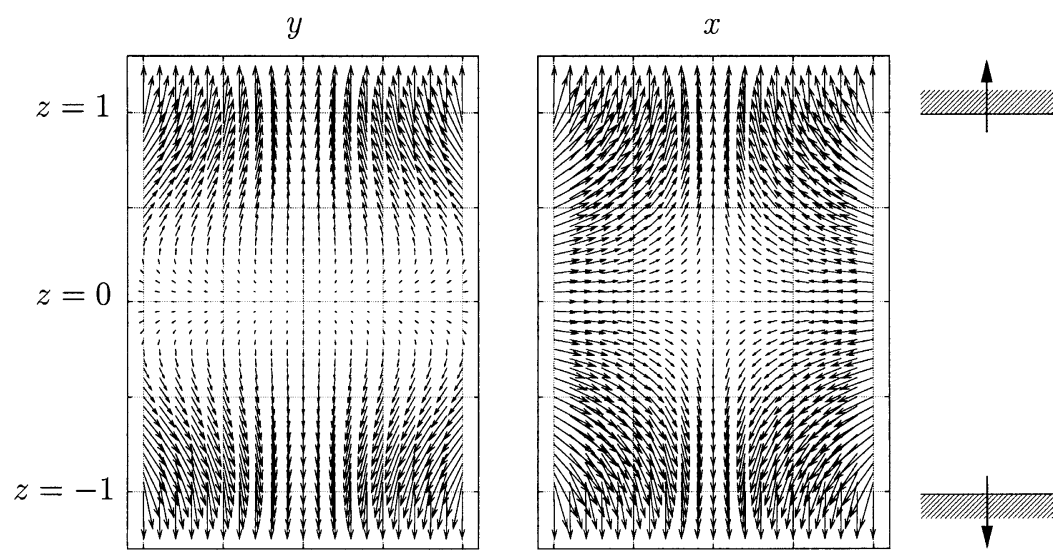

Fig. 7. The flow field in the $x-z$ and $y-z$ planes for a non-zero $F$ solution obtained at $\mu=1 / 2$ and $R e=2$. Note that the mass flux in the $x$-direction is three times that in the $y$-direction when $\mu=1 / 2$.

states following a secondary instability through a supercritical Hopf bifurcation. We shall discuss an initial-value formulation of this problem in later sections of this work; however, here we may simply demonstrate the supercritical nature of the periodic states by the phase-plane plot of Fig. 8 .

At the arbitrarily chosen values of $\mu=1 / 2, \operatorname{Re}=2$ and $\delta=-1$, together with symmetric suction applied at both walls $(\gamma=1)$, the flow field in the mutually perpendicular planes $x-z$ and $y-z$ is shown in Fig. 7. It is easily seen that in this case there are regions of outward and inward flow, although the inward flow is obviously dominant since the boundary transpiration acts to withdraw fluid. Furthermore, to illustrate the swirling nature of the flow, the three-dimensional streamlines originating from a sequence of points in the plane $z=-0.2$ are also shown in Fig. 9.

We may furthermore note that the ellipticity parameter $c$ in formulation (6) can effectively be set to unity, since for any given $c$, the entirety of the solution set can be spanned by variation of 


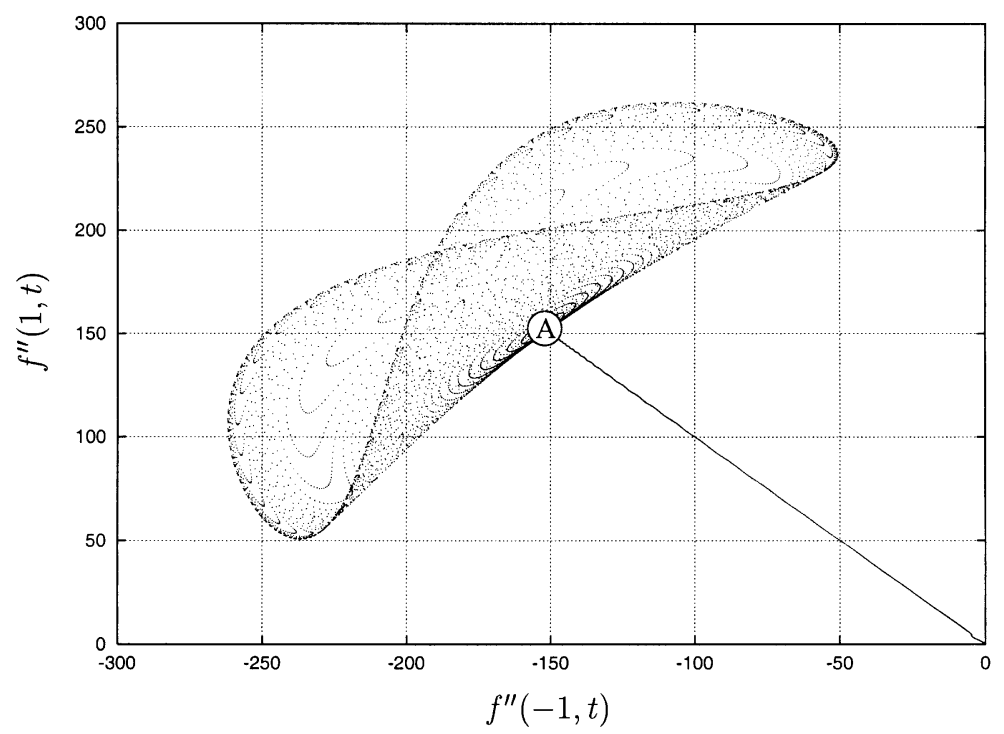

Fig. 8. An evolution of the unsteady boundary-value problem to a limit cycle solution (arising through a Hopf bifurcation) at $\mu=0, R e=10$. Point A denotes the location of the steady solution in this phase plane, corresponding to the axisymmetric "bifurcation to swirl" state as described by Goldshtik and Javorsky (1989).

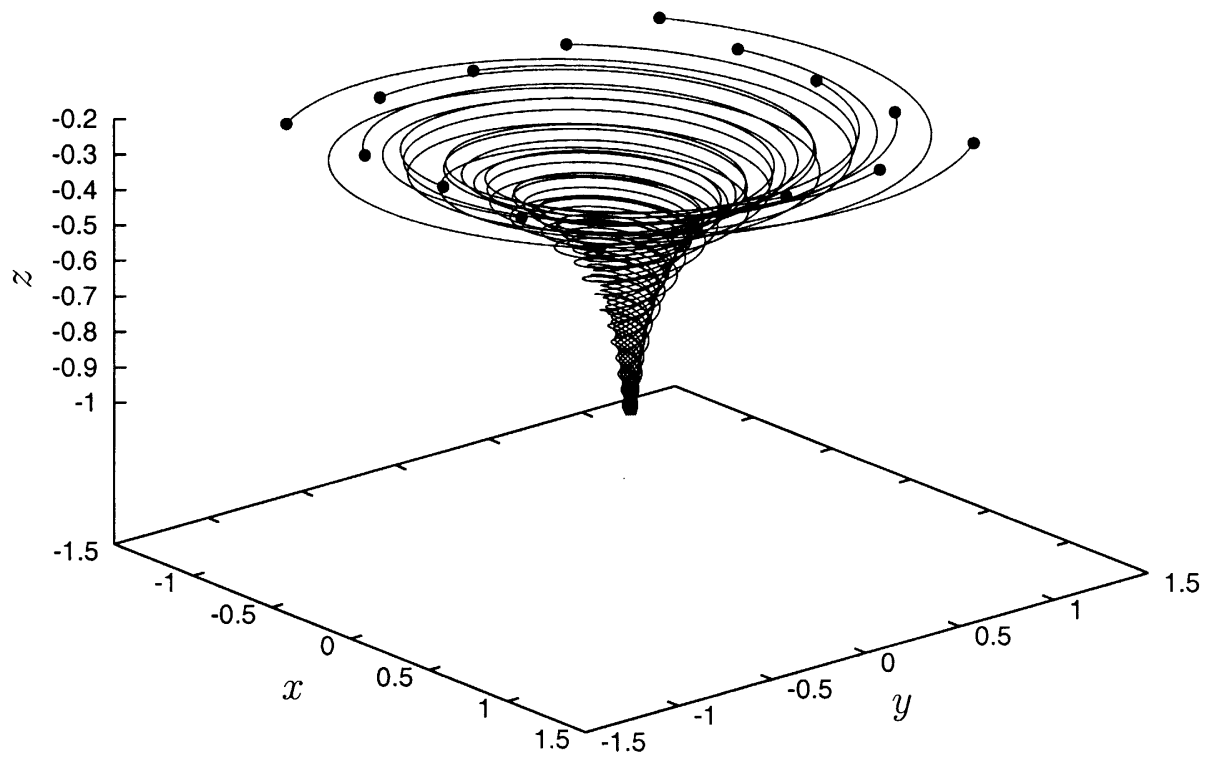

Fig. 9. The streamlines for a non-zero $F$ solution obtained at $\mu=1 / 2$ and $R e=2$. This pattern of streamlines is repeated (but inverted) in the upper half of the domain since the solution is symmetric about the midplane. The streamlines shown originate from a square of starting points (as illustrated by the circular points) in the plane $z=-0.2$. 


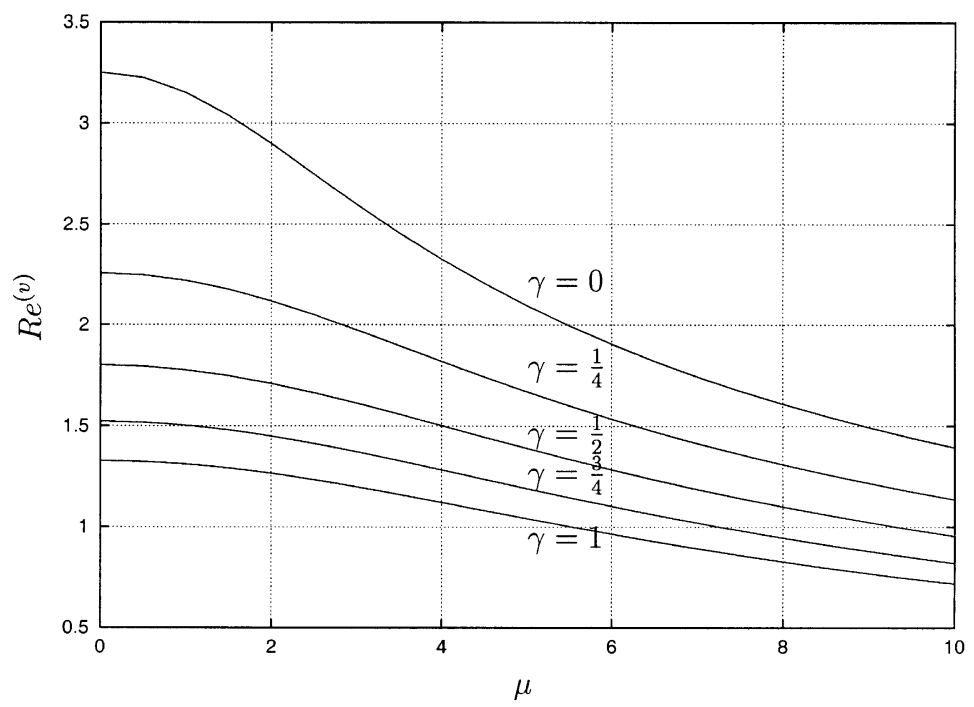

Fig. 10. Location of pitchfork bifurcation from states from $F \equiv 0$ to $F \neq 0$ in the case $\delta=-1$.

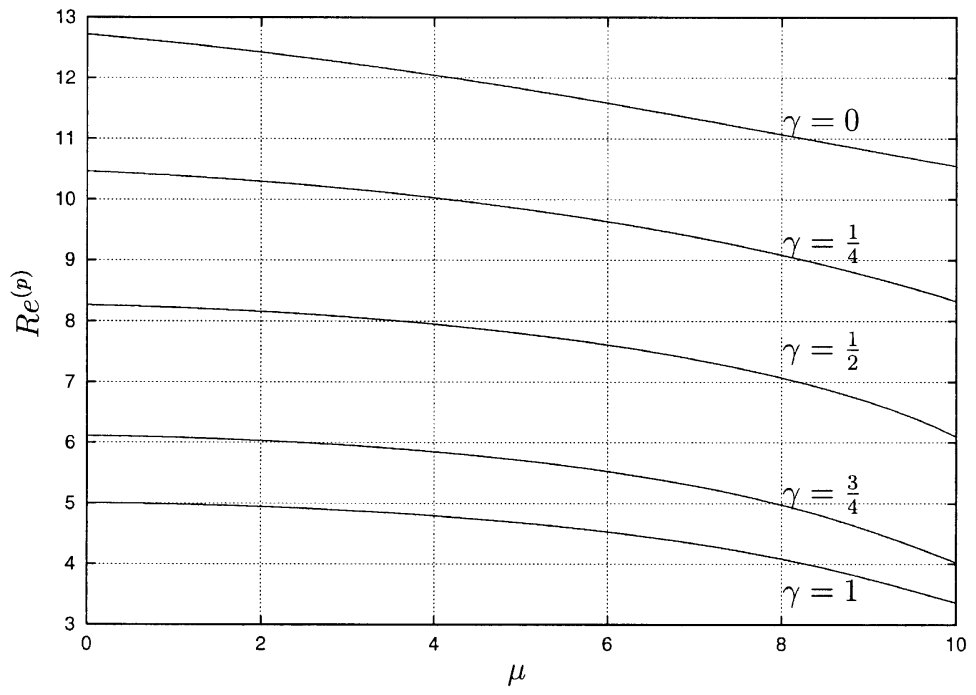

Fig. 11. Location of Hopf bifurcation from states with $F \neq 0$ in the case $\delta=-1$.

$\mu$ alone. This is again evident from the cylindrical polar formulation, since the constant $c$ acts to simply alter the relative magnitudes of $A$ and $B$.

Fig. 10 shows the location of the critical Reynolds number $\left(R e^{(v)}\right)$ at which the $F \neq 0$ states bifurcate from the $F \equiv 0$ states.

The location of the aforementioned Hopf bifurcation is also of some interest. Fig. 11 shows how variations in the wall parameter $\mu$ (and $\gamma$ ) affect the critical Reynolds number $R e^{(\mathrm{p})}$. Note that (in 


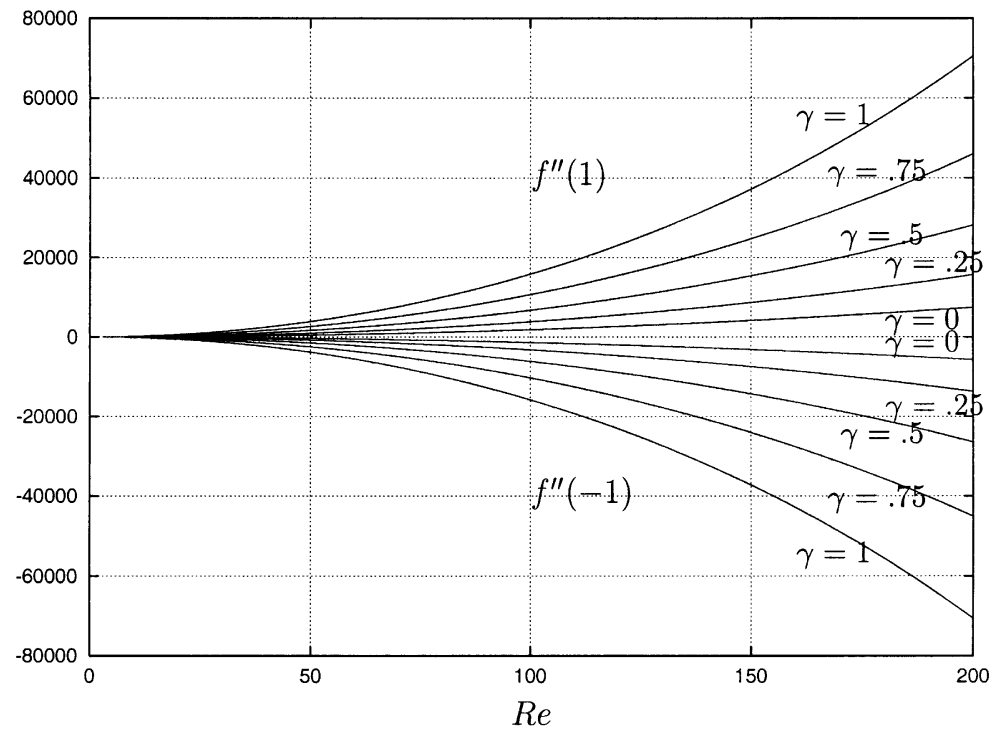

Fig. 12. Variation of $f^{\prime \prime}( \pm 1)$ with $\operatorname{Re}$ at $\gamma$ values as shown, $\mu=1, \delta=-1$.

comparison with Fig. 10) for a particular solution branch/fixed value of the parameter $\gamma$, the Hopf bifurcation seems always to occur at a higher Reynolds number than the pitchfork bifurcation and on the $F \neq 0$ state.

\subsection{The large Reynolds number limit}

Here, we consider the solution structure in the limit of large Reynolds numbers. Results for the variation of $f^{\prime \prime}( \pm 1)$ and $F^{\prime}( \pm 1)$ with Reynolds number (with, crucially $\left.\delta=-1\right)$ for selected values of the suction/injection parameter $\gamma$ and $\mu=1$ are shown in Figs. 12 and 13, respectively. We note that the corresponding figure for $g^{\prime \prime}( \pm 1)$ is similar to Fig. 12; the difference between these two quantities is in fact $O(1)$ but is dominated by the larger leading-order behaviour. These figures strongly suggest that as the Reynolds number becomes large, all the aforementioned flow variables become very large. Inspection of the solution profiles (see Fig. 14 showing results at $R e=50,60,70,80,90,100$ - highest values exhibiting the largest gradients near the walls) clearly indicates also that for the most part (i.e., away from the channel walls): (i) $F(z)$ becomes increasingly independent of $z$ and is clearly seen to scale linearly with $\operatorname{Re}$ and (ii) both $f(z)$ and $g(z)$ are linear functions of $z$ and $O(1)$. Guided by these observations, the conjecture is then therefore that the following scalings are appropriate, as $R e \rightarrow \infty$, away from the walls

$$
\left(f, g, F, K_{1}, K_{2}\right)=\left(f_{0}(z), g_{0}(z), \operatorname{ReF}_{0}(z), \operatorname{Re}^{2} K_{10}, \operatorname{Re}^{2} K_{20}\right)+\text { h.o.t., }
$$

which, upon substitution into (7c) and (20b) leads to the conclusions that

$$
\begin{aligned}
& f_{0}=\Lambda_{0} z+\Lambda_{1}, \\
& g_{0}=-\Lambda_{0} z+\Lambda_{2},
\end{aligned}
$$




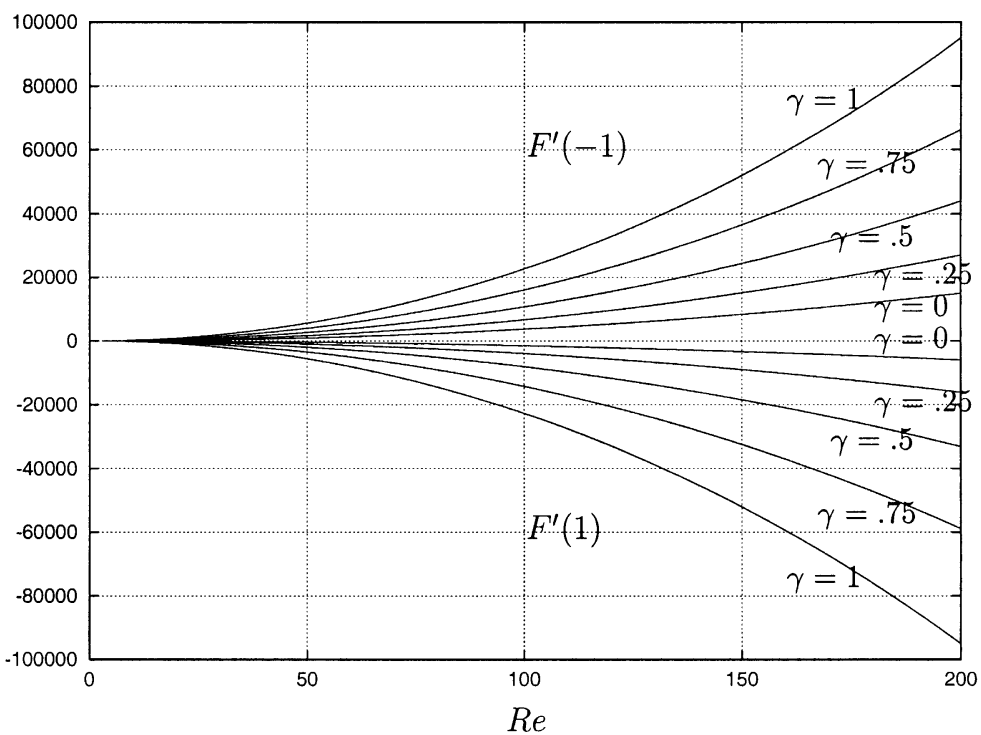

Fig. 13. Variation of $F^{\prime}( \pm 1)$ with $R e$ at $\gamma$ values as shown, $\mu=1, \delta=-1$.

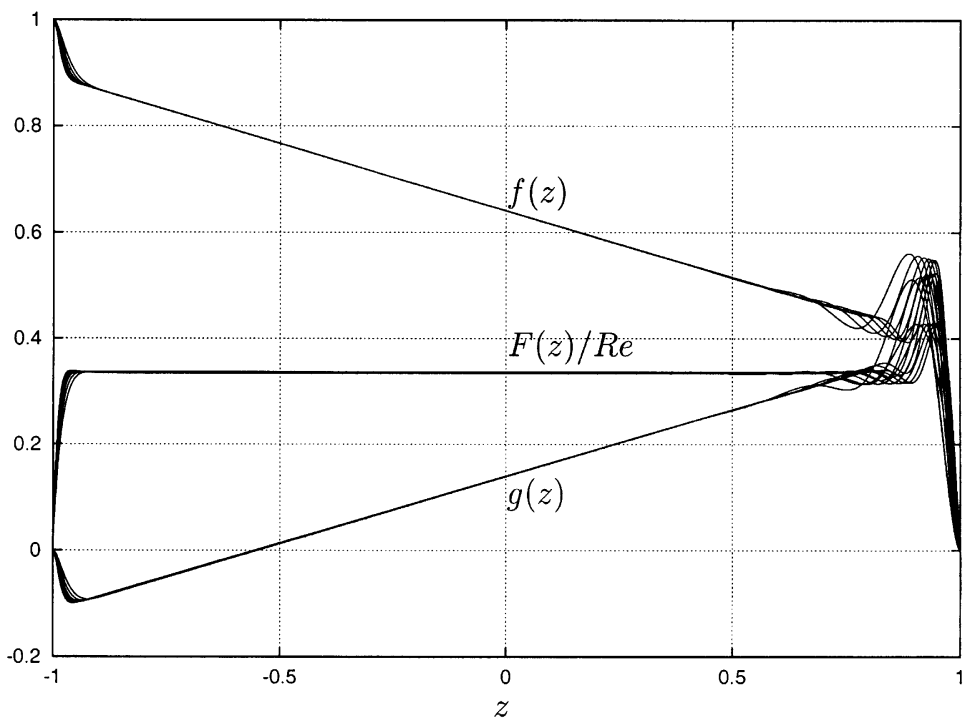

Fig. 14. Solution profiles for $\mu=1, \gamma=0, R e=50,60,70,80,90,100$ (larger values of $R e$ exhibit larger solution gradients near boundaries).

$$
\begin{aligned}
& K_{10}=K_{20}, \\
& F_{0}=K_{10}^{1 / 2}=K_{20}^{1 / 2},
\end{aligned}
$$

where $\Lambda_{0}, \Lambda_{1}$ and $\Lambda_{2}$ are all constants. Here note in particular how $F_{0}$ is essentially independent of $z$, whilst both $f_{0}$ and $g_{0}$ are linear in $z$, with equal and opposite gradients. 
The wall layers have a non-dimensional thickness $O\left(R e^{-1}\right)$; considering the lower wall layer, the appropriate scalings are then

$$
(f, g, F)=\left(f^{-}\left(Z^{-}\right), g^{-}\left(Z^{-}\right), \operatorname{ReF}^{-}\left(Z^{-}\right)\right)+\text {h.o.t., }
$$

where $Z^{-}=(z+1) R e$ defines the boundary-layer scale. The governing equations are then

$$
\begin{aligned}
& f_{Z^{-} Z^{-} Z^{-}}^{-}+\left(f^{-}+g^{-}\right) f_{Z^{-} Z^{-}}^{-}-\left(f_{Z^{-}}^{-}\right)^{2}+\left(F^{-}\right)^{2}=K_{10}, \\
& g_{Z^{-} Z^{-} Z^{-}}^{-}+\left(f^{-}+g^{-}\right) g_{Z^{-} Z^{-}}^{-}-\left(g_{Z^{-}}^{-}\right)^{2}+\left(F^{-}\right)^{2}=K_{10}, \\
& F_{Z^{-} Z^{-}}^{-}-\left(f_{Z^{-}}^{-}+g_{Z^{-}}^{-}\right) F^{-}+\left(f^{-}+g^{-}\right) F_{Z^{-}}^{-}=0,
\end{aligned}
$$

subject to $f^{-}(0)=\frac{1}{2}(1+\mu), g^{-}(0)=\frac{1}{2}(1-\mu), F^{-}(0)=f_{Z^{-}}^{-}(0)=g_{Z^{-}}^{-}(0)=0, F^{-} \rightarrow K_{10}^{1 / 2}, f_{Z^{-}}^{-}, g_{Z^{-}}^{-} \rightarrow$ 0 as $Z^{-} \rightarrow \infty$. The upper wall layer exhibits the same basic structure, with variables (in our notation $f^{+}\left(Z^{+}\right), g^{+}\left(Z^{+}\right), F^{+}\left(Z^{+}\right)$, where $Z^{+}=(Z-1) R e$, with the boundary conditions changed in an obvious manner.

The key point now is that the two wall layers must both be compatible with the core solution (28), which in particular demands that

$$
f^{-}\left(Z^{-} \rightarrow \infty\right)+g^{-}\left(Z^{-} \rightarrow \infty\right)=f^{+}\left(Z^{+} \rightarrow-\infty\right)+g^{+}\left(Z^{+} \rightarrow-\infty\right)
$$

It is this condition that serves to determine the key quantity $K_{10}^{1 / 2}$, this being the (constant) value of $F(z)$ in the core. Fig. 15 shows the results of solving the above system (with the value of $\left(K_{10}+\Lambda_{0}^{2}\right)^{1 / 2}$ appropriately chosen) for the case of $\mu=1, \gamma=0$. It should be noted that for the

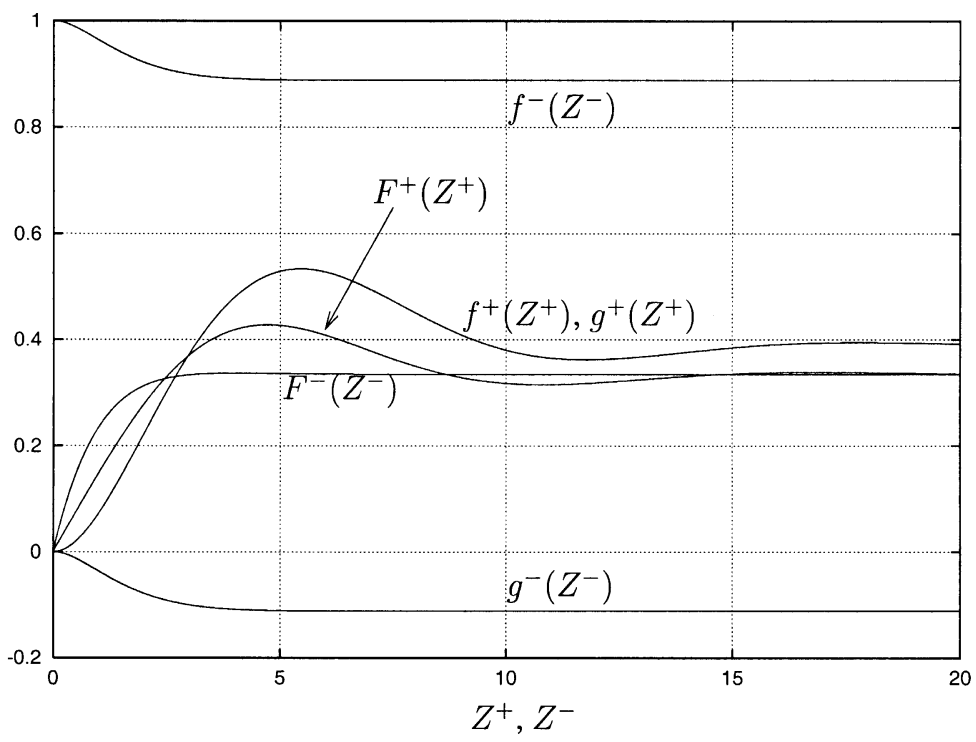

Fig. 15. Asymptotic solution profiles (near boundaries). 
case $\gamma=0, f^{+}\left(Z^{+}\right) \equiv g^{+}\left(Z^{+}\right)$. These results are to be compared with those shown in Fig. 14, in particular, close to $z= \pm 1$. Note also the especially good comparison between our asymptotic results using (30) which predict $K_{10}^{1 / 2} \approx 0.3346$ and the almost constant core values of $F(z) / R e$ at increasing $\operatorname{Re}$ (using the 'full system'), as shown in Fig. 14.

\section{Unsteady calculations}

We now consider the nature of the flow, using an initial-value-type of approach to the problem, with particular attention to flow parameter regimes for which no steady-state solutions exist. For the choice of parameters to be shown, the nature of the flow development differs fundamentally according to the value of $\delta$. In the case of $\delta=-1$, steady-state solutions were attained in all the calculations performed in the parameter range of interest using the steady equations, and will not be considered in this section); however, for $\delta=0$ or 1 it was apparent that the solutions frequently grew significantly in amplitude, and we focus our attention on this aspect of the flow in this section.

In the case $\delta=0$, in regimes for which no steady-state solutions existed (i.e., $\mu>\mu_{\mathrm{c}}$ ), very large solution amplitudes were obtained. In some cases it appeared that a singularity might be forming, or alternatively large unbounded growth (in time) was occurring. It is notable that much of the analysis of the $\mu \rightarrow \mu_{\mathrm{c}}$ limit of Section 3 is again applicable, but with the large parameter $1 /\left(\mu_{\mathrm{c}}-\mu\right)$ replaced by $\kappa(t)$, where $|\kappa| \gg 1$. Indeed, the solution behaviour (in particular, the solution profiles $f(z)$ and $g(z)$ resembled very much those shown in Figs. 4 and 5). Although a finite-time singularity cannot be dismissed as a possible scenario, it is worth mentioning that Cox (1991) showed that in the case of the Berman (1953) problem (in our terminology, the case $g=F=0$ ), the solution does persist without breakdown, in contrast to the suggestion of Childress et al. (1989). Subsequently King and Cox (2001) went on to show (using an "exact" solution to the Berman problem) the nature of these persisting solutions. It is interesting, in this context, to note that the analysis of King and Cox (2001) can be extended to our case for which $g \leqslant 0$ (but with $F=0$ ) as follows. It turns out that a solution of the form

$$
\begin{aligned}
& f=A_{0}(t)+B_{0}(t) z+C_{0}(t) \sin (\lambda(t) z)+D_{0}(t) \cos (\lambda(t) z), \\
& g=A_{1}(t)+B_{1}(t) z
\end{aligned}
$$

admits an exact solution to (7a) and (7b) provided three conditions linking the $A_{0}(t), A_{1}(t), B_{0}(t)$, $B_{1}(t), C_{0}(t), D_{0}(t)$ and $\lambda(t)$ are satisfied (whilst other conditions may be provided by the wall conditions), in a self-consistent manner, analogous to that employed by King and Cox (2001).

We now move on to consider a computation with $\delta=1$ in which $F(z, t)$ was explicitly triggered through the introduction of an "imperfection" in the (lower) wall boundary condition. The specific calculation performed was with

$$
\begin{aligned}
& f(-1, t)=\frac{1}{2}\left(1-e^{-t}\right)^{4}(1+\mu), \quad g(-1, t)=\frac{1}{2}\left(1-e^{-t}\right)^{4}(1-\mu), \\
& f(1, t)=-\frac{\gamma}{2}\left(1-e^{-t}\right)^{4}(1+\mu), \quad g(1, t)=-\frac{\gamma}{2}\left(1-e^{-t}\right)^{4}(1-\mu), \\
& F(-1, t)=\varepsilon t^{4} e^{-t},
\end{aligned}
$$




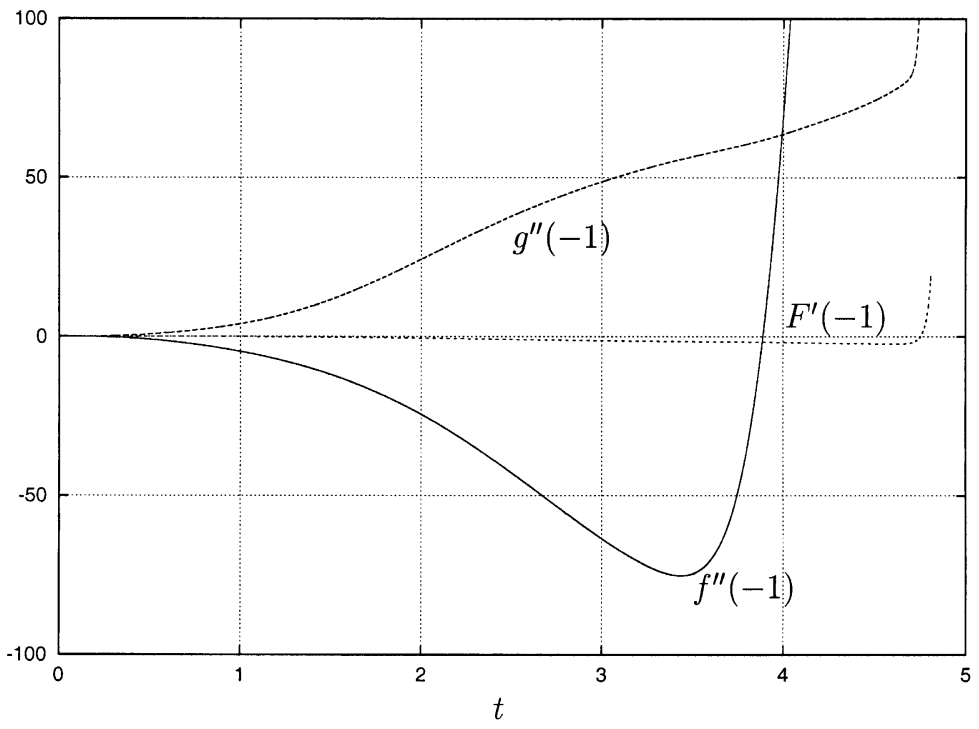

Fig. 16. Temporal development of $f^{\prime \prime}(-1), g^{\prime \prime}(-1)$ and $F^{\prime}(-1) ; \mu=7.5, \gamma=0, R e=40, \delta=1$.

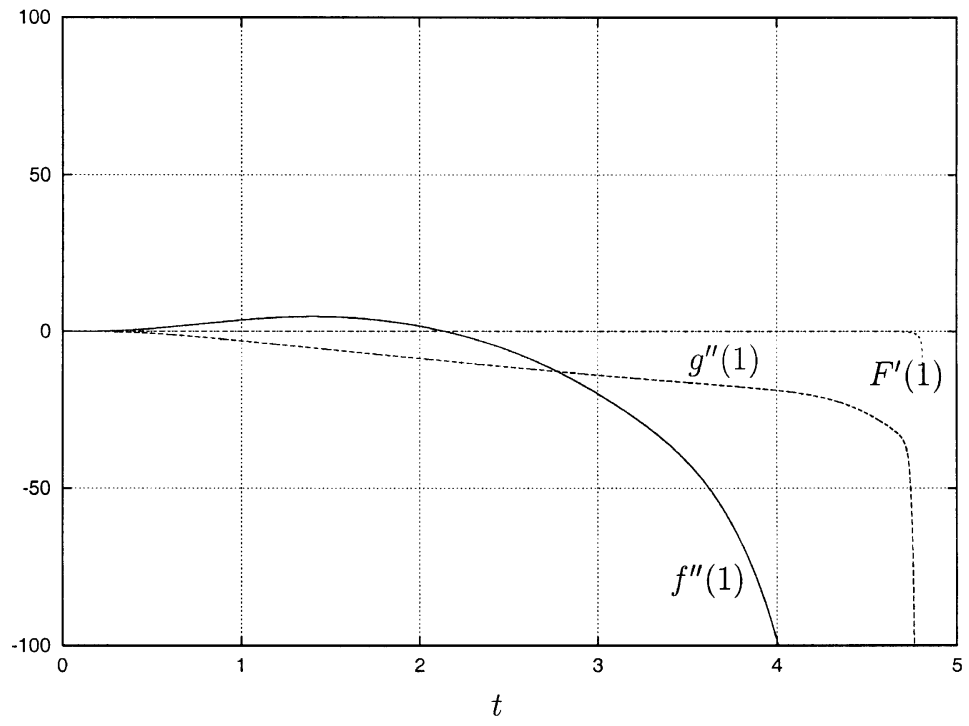

Fig. 17. Temporal development of $f^{\prime \prime}(1), g^{\prime \prime}(1)$ and $F^{\prime}(1) ; \mu=7.5, \gamma=0, R e=40, \delta=1$.

where in the results to be presented, $\mu=7.5, \gamma=0$ (this is akin to the Cox, 2002 problem, but with $F(z, t)), \varepsilon=0.01$ and $R e=40$; the homogeneous upper wall boundary condition on $F$ was retained.

Results for wall values of the solution are presented in Figs. 16 (lower wall) and 17 (upper wall) and it is quite clear from these results that a finite-time singularity is being approached (somewhat sooner than in the corresponding $\delta=0$ case). Away from the channel walls, the following asymptotic 


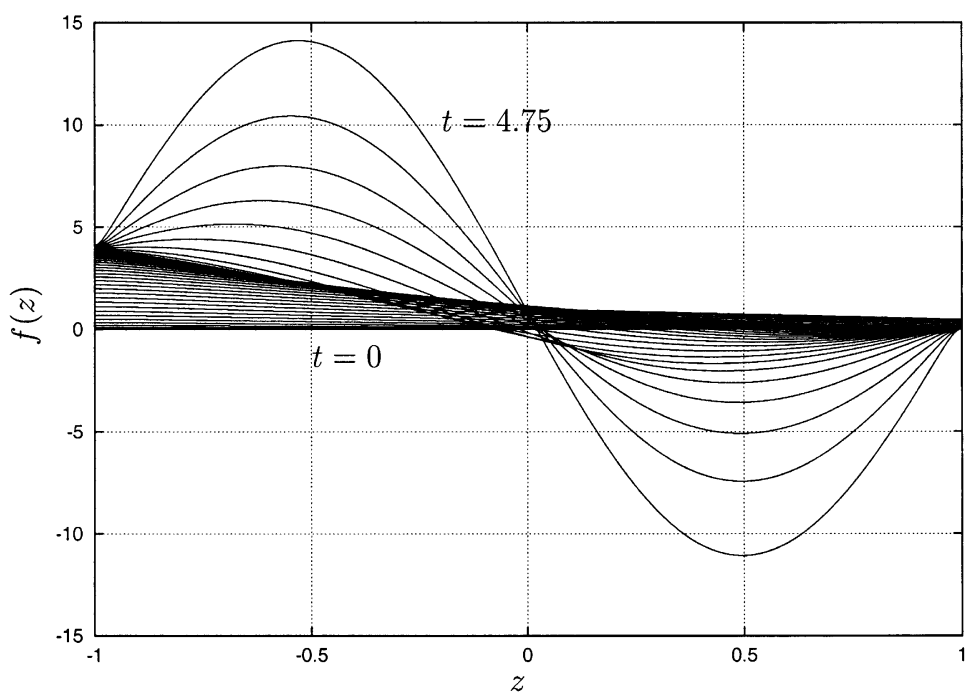

Fig. 18. $f(z)$ profiles, $\mu=7.5, \gamma=0, R e=40, \delta=1$.

expansions are the most appropriate:

$$
\begin{aligned}
& f=\left(t_{\mathrm{s}}-t\right)^{-1} f_{0}(z)+\cdots, \quad g=\left(t_{\mathrm{s}}-t\right)^{-1} g_{0}(z)+\cdots, \quad F=\left(t_{\mathrm{s}}-t\right)^{-1} F_{0}(z)+\cdots, \\
& K_{1}=\left(t_{\mathrm{s}}-t\right)^{-2} K_{10}+\cdots, \quad K_{2}=\left(t_{\mathrm{s}}-t\right)^{-2} K_{20}+\cdots .
\end{aligned}
$$

Substituting this into (20b) (with the addition of the time-derivative term in this system) leads to the following system of equations:

$$
\begin{aligned}
& -f_{0}^{\prime}-f_{0}^{\prime 2}+\left(f_{0}+g_{0}\right) f_{0}^{\prime \prime}+F_{0}^{2}=K_{10}, \\
& -g_{0}^{\prime}-g_{0}^{\prime 2}+\left(f_{0}+g_{0}\right) g_{0}^{\prime \prime}+F_{0}^{2}=K_{20}, \\
& F_{0}+F_{0} g_{0}^{\prime}-g_{0} F_{0}^{\prime}=0,
\end{aligned}
$$

subject to $f( \pm 1)=g( \pm 1)=F( \pm 1)=0$. Indeed, a similar system has been found in related problems studied by Hall et al. (1992) and Al-Azhari (2003). Solutions (which are strongly suggested in the profile results shown in Figs. 18, 19 and 20) may be sought in the form

$$
\begin{aligned}
& f_{0}(z)=\alpha_{0} \sin (n \pi z), \\
& g_{0}(z)=\beta_{0} \sin (n \pi z), \\
& F_{0}(z)=\gamma_{0}[1+\cos (n \pi z)] .
\end{aligned}
$$




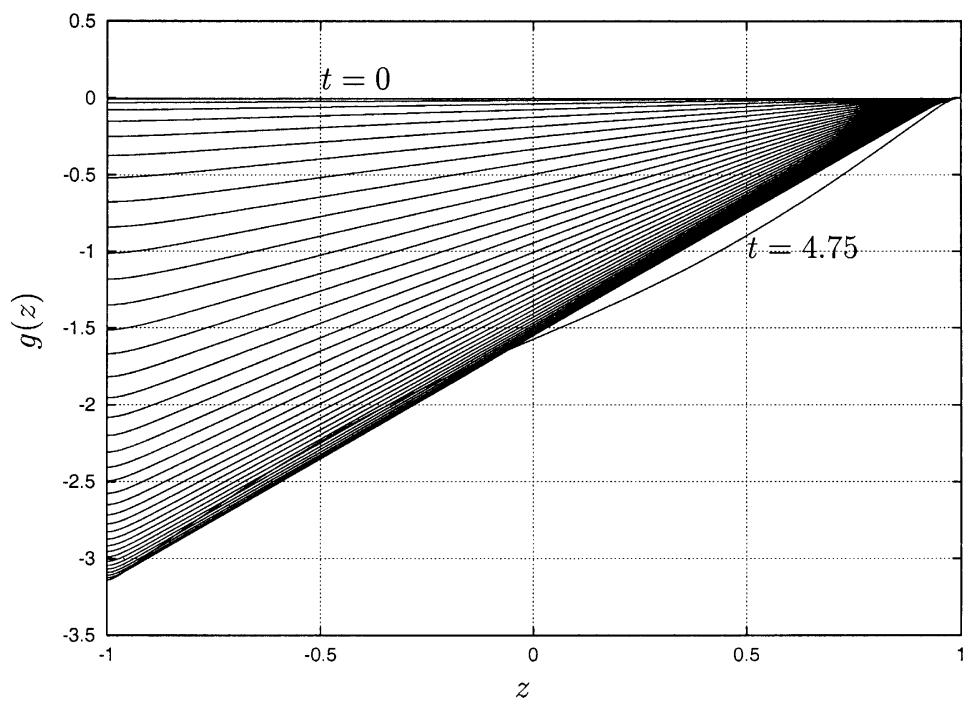

Fig. 19. $g(z)$ profiles, $\mu=7.5, \gamma=0, R e=40, \delta=1$.

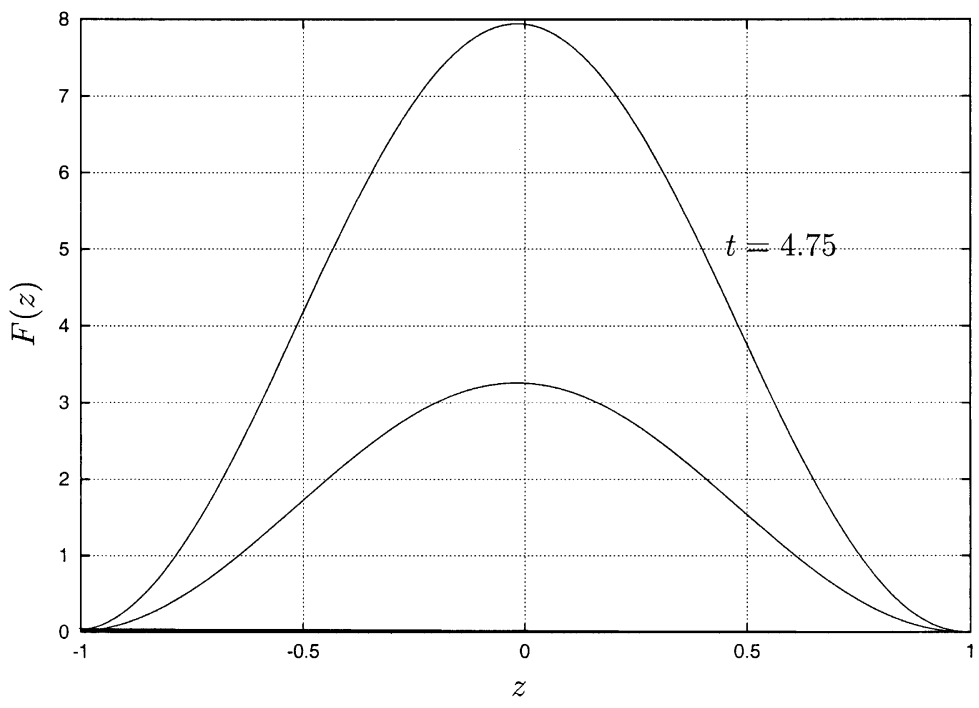

Fig. 20. $F(z)$ profiles, $\mu=7.5, \gamma=0, \operatorname{Re}=40, \delta=1$.

Substituting these forms into (37)-(39) leads to the conditions:

$$
\alpha_{0}=\beta_{0}=-\frac{1}{2 n \pi} n=1,2,3 \ldots, \quad \gamma_{0}=\frac{1}{2}, \quad K_{10}=K_{20}=-\frac{3}{4} .
$$

Although in the computations performed for this study all clearly pointed to the case $n=1$, other computations performed in the related study of Al-Azhari (2003) certainly indicate that is not always the case. 
It has been shown by Al-Azhari (2003) that the boundary layers that form in this case are of thickness $O\left(\left(t_{\mathrm{s}}-t\right)^{1 / 2}\right)$; it is also worth noting that if $n \neq 1$ above, then the flow (formally) comprises a sequence of inviscid cells, separated by $O\left(\left(t_{\mathrm{s}}-t\right)^{1 / 2}\right)$ viscous layers.

\section{Discussion}

In this work, we have reconsidered a range of self-similar flows between two infinite, parallel, porous planes. Many results for such flows have been provided by Philip Drazin and his collaborators, and here we have extended them further. The class of stagnation-point solutions that we seek are well known in many contexts, including flows of rotating-disk type, and of boundary-layer form.

We have taken the three-dimensional solutions provided by Taylor et al. (1991) and extended the solution structure to include an additional component (denoted by $F(z)$ herein). The inclusion of this new term allows for the inclusion of swirl. It is easy to note that the axial component of the vorticity for this type of self-similar flow is $c^{-1 / 2}(\delta c-1) F(z)$. So we can observe that, provided that $\delta c \neq 1$, the flow character is fundamentally altered. We can also highlight that in the case $\delta=-1, c=1$, the solution structure provided by (6) is equivalent to that provided by Goldshtik and Javorsky (1989); here the cylindrical-polar coordinate description of their paper has been resolved into its Cartesian equivalent in our description. However, the solution of Goldshtik and Javorsky is axisymmetric, whereas here the solution is such that the mass transport in the in-plane directions (i.e., parallel to the $x$ - and $y$-axis) may be chosen independently and the parameter $c$ can be viewed as a measure of flow ellipticity. Given the form of solution, it is evident that the ellipticity parameter $c$ can be specified arbitrarily. We thus see that the solutions of Goldshtik and Javorsky are generic and can be extended to flows that behave in a qualitatively similar manner; with the generation of swirl through a bifurcation, followed by the appearance of periodic states following a Hopf bifurcation arising from the nonlinear solution with non-zero axial vorticity.

Another interesting feature of this system is the dependence of the solution structure on the assumption of fixed relative mass flux versus fixed relative pressure dependence. In particular, in formulating the steady boundary-value problem one must either specify the relative mass flux parameter $\mu$ or the pressure ratio $K_{1} / K_{2}$. These two formulations are distinct however. For example, on specifying that $K_{1}=K_{2}$, the non-axisymmetric state shown in Fig. 1 (with $F=0$ ) arises through a sub-critical pitchfork bifurcation, and thus in the similarly formulated initial-value problem, the non-axisymmetric state cannot be realised through a time-dependent process owing to an instability. However, a formulation of the problem in terms of a fixed relative mass flux reveals a full one-parameter family of solutions, spanned by $\mu$, at a fixed Reynolds number, some of which will have (in addition) $K_{1}=K_{2}$. In this latter context, the non-axisymmetric solution branch is (in general) achievable in the initial-value problem. One might find these two viewpoints surprising in that the two conditions can essentially be viewed as fixing the flow in the far-field, but lead to distinct evolutions of the self-similar flow. This is in contrast to the usual assumptions regarding the applicability of self-similar solutions and their adjustment to meet appropriate boundary conditions via some edge region of limited influence in a finite geometry. The possibility of solutions of this type providing a basis for a more detailed analysis of the applicability of self-similar solutions and the influence of far-field conditions in large aspect-ratio domains is a subject for future work. It is also noteworthy that steady-state solutions with $\delta=-1$ do/can exist where steady-state solutions with $\delta=0$ or 1 do not. 


\section{Acknowledgements}

During his long and productive career, Philip Drazin and his collaborators produced many important papers on the subjects of internal flows and exact solutions to the Navier-Stokes equations (amongst others). Much of the work concentrated on clarifying the structure of steady, periodic (and in some cases, chaotic) states in such flows and consideration of their stability properties. The authors (REH and PWD) of this current paper, benefited on a number of occasions from Philip's insight and broad knowledge of fluid mechanics, self-similar flows and, in particular, exact solutions of the Navier-Stokes system.

The authors wish to thank the anonymous referees, who pointed out a number of shortcomings in the original version of the paper.

\section{References}

Al-Azhari, 2003. Ph.D thesis, University of Manchester. On the flow between two rotating disks and related problems. Berman, A.S., 1953. Laminar flow in channels with porous walls. J. Appl. Phys. 24, 1232.

Childress, S., Ierley, G.R., Spiegel, E.A., Young, W.R., 1989. Blow-up of unsteady two-dimensional Euler and NavierStokes solutions having stagnation-point form. J. Fluid Mech. 203, 1.

Cox, S.M., 1991. Two-dimensional flow of a viscous fluid in a channel with porous walls. J. Fluid Mech. $227,1$.

Cox, S.M., 2002. Non-axisymmetric flow between an air table and a floating disk. Phys. Fluids 14, 1540.

Cox, S.M., King, A.C., 1997. On the asymptotic solution of a high-order nonlinear ordinary differential equation. Proc. R. Soc. London A 453, 711.

Davey, A., Schofield, D., 1967. Three-dimensional flow near a two-dimensional stagnation point. J. Fluid Mech. $28,149$. Goldshtik, M.A., Javorsky, N.J., 1989. On the flow between a porous rotating disk and a plane. J. Fluid Mech. 207 , 1.

Hall, P., Balakumar, P., Papageorgiou, D., 1992. On a class of unsteady three-dimensional Navier-Stokes solutions relevant to rotating-disc flows: threshold amplitudes and finite-time singularities. J. Fluid Mech. 238, 297.

Hewitt, R.E., Duck, P.W., 2000. Non-axisymmetric rotating-disk flows: nonlinear travelling-wave states. J. Fluid Mech. 413, 287.

Hewitt, R.E., Duck, P.W., Stow, S., 2002. Continua of states in boundary-layer flows. J. Fluid Mech. $468,121$.

Hiemenz, K., 1911. Die Grenzschicht an einem in den gleichförmigen Flüssigkeitsstrom eingetauchten geraden Kreiszylinder. Dinglers J. 326, 321.

Hinch, E.J., Lemaitre, J., 1994. The effect of viscosity on the height of disks floating above an air table. J. Fluid Mech. $273,313$.

Homann, F., 1936. Der Einfluss grosser Zähigkeit bei der Strömung um den Zylinder und um die Kugel. Z. Angew. Math. Mech. 16, 153.

Howarth, L., 1934. On calculation of the steady flow in the boundary layer near the surface of a cylinder in a stream. Reports and memoranda-Aeronautical research council London No. 1632.

King, J.R., Cox, S.M., 2001. Asymptotic analysis of the steady-state and time-dependent Berman problem. J. Eng. Math. $39,87$.

Taylor, C.L., Banks, W.H.H., Zaturska, M.B., Drazin, P.G., 1991. Three-dimensional flow in a porous channel. Quart. J. Mech. Appl. Math. 44, 105.

Watson, E.B.B., Banks, W.H.H., Zaturska, M.B., Drazin, P.G., 1990. On transition to chaos in a two-dimensional channel flow symmetrically driven by accelerating walls. J. Fluid Mech. 212, 451.

Zaturska, M.B., Drazin, P.G., Banks, W.H.H., 1988. On the flow of a viscous fluid driven along a channel by suction at porous walls. Fluid Dyn. Res. 4, 151. 